PROCEEDINGS OF THE

AMERICAN MATHEMATICAL SOCIETY

Volume 136, Number 1, January 2008, Pages 161-170

S 0002-9939(07)08936-8

Article electronically published on September 25, 2007

\title{
BLASCHKE REPRESENTATION OF FUNCTIONS ON THE CIRCLE
}

\author{
ELIAS WEGERT AND LOTHAR VON WOLFERSDORF
}

(Communicated by Joseph A. Ball)

\begin{abstract}
We prove that every unimodularly bounded measurable function on the complex unit circle admits a representation

$$
f=\frac{f_{+}+f_{-}}{1+\bar{f}_{-} f_{+}}
$$

where $f_{+}$and $f_{-}$extend holomorphically into the interior and the exterior of the circle, respectively, $f_{-}$vanishes at infinity, and both functions are unimodularly bounded. The representation is unique if $\|f\|_{\infty}<1$.
\end{abstract}

Representations of functions on the complex unit circle $\mathbb{T}$ by boundary values of holomorphic functions have been known for a long time and play an important rôle in theoretical investigations as well as in applications.

One of the two most popular representations of this type is the additive Riesz decomposition

$$
f=f_{+}+f_{-},
$$

where $f_{+}$and $f_{-}$are functions which extend holomorphically into the interior $\mathbb{D}$ and the exterior $\mathbb{E}:=\mathbb{C} \backslash \overline{\mathbb{D}}$ of the unit circle, respectively, and $f_{-}$vanishes at infinity. Its multiplicative counterpart is the Wiener-Hopf factorization,

$$
f=f_{+} \cdot f_{\kappa} \cdot f_{-},
$$

where $f_{+}$and $f_{-}$are additionally supposed to be nonvanishing in their domains, $f_{-}(\infty)=1$, and $f_{\kappa}(t)=t^{\kappa}$ with $\kappa \in \mathbb{Z}$.

This paper is devoted to another representation which fits into this framework, and appears, to our knowledge, for the first time in the paper [6] by J. Sylvester and D. Winebrenner. Investigating inverse scattering in stratified media, they introduced a nonlinear decomposition of the form

$$
f=f_{-} \circ f_{+}:=\frac{f_{+}+f_{-}}{1+\bar{f}_{-} f_{+}}
$$

for functions $f$ on the real line, where $f_{+}$and $f_{-}$are holomorphic in the upper and lower half-plane, respectively. Sylvester and Winebrenner proved existence and uniqueness of this representation in the case where $f$ is representable as a Fourier transform of a function supported in the interval $(-a,+\infty)$ with $a>0$ and $-\int \log \left(1-|f|^{2}\right)<\pi /(2 a)$ (see Theorem 2.5 in [6]).

Received by the editors June 26, 2006 and, in revised form, September 22, 2006.

2000 Mathematics Subject Classification. Primary 30E25; Secondary 30D50, 81U40.

(C) 2007 American Mathematical Society 
The goal of this paper is to show that in fact every unimodularly bounded measurable function $f$ on $\mathbb{T}$ admits a representation of the form (0.3) with unimodularly bounded holomorphic functions $f_{+}$and $f_{-}$in $\mathbb{D}$ and $\mathbb{E}$, respectively. Since the righthand side of (0.3) has the form of a Blaschke factor we propose the name Blaschke representation of $f$. One may think of it as a hyperbolic counterpart of the Riesz decomposition. This is supported by the fact that $|w|=1$ is a natural bound for the values of all involved functions.

We remark that substituting $\bar{f}_{-}$by $f_{-}$in the denominator of (0.3) would yield a special case of a so-called linear fractional conjugation boundary value problem investigated by Mityushev and Rogosin (Section 3.6 of [4]). However, the nice properties of the Blaschke representation hinge exactly on this complex conjugation.

In order to give a precise statement we denote by $L^{p}(\mathbb{T})$ (or $L^{p}$ for short), with $1 \leq p \leq \infty$, the Lebesgue spaces of measurable functions on $\mathbb{T}$ with norm $\|\cdot\|_{p}$. The Hardy spaces of holomorphic functions in $\mathbb{D}$ are denoted by $H_{+}^{p}$, while $H_{-}^{p}$ stands for the corresponding Hardy spaces of holomorphic functions in $\mathbb{E}$ which vanish at infinity. As usual, functions in $H_{ \pm}^{p}$ are identified with their boundary functions and the Hardy spaces $H_{ \pm}^{p}$ are considered as subspaces of $L^{p}$.

Theorem 0.1. Let $f \in L^{\infty}$ be a bounded measurable function on $\mathbb{T}$ with $\|f\|_{\infty} \leq 1$.

(i) There exist functions $f_{+} \in H_{+}^{\infty}$ and $f_{-} \in H_{-}^{\infty}$ with $\left\|f_{ \pm}\right\|_{\infty} \leq 1$ such that almost everywhere on $\mathbb{T}$,

$$
f=f_{-} \circ f_{+}:=\frac{f_{+}+f_{-}}{1+\bar{f}_{-} f_{+}} .
$$

(ii) If $\|f\|_{\infty}<1$, then the functions $f_{ \pm}$in the representation (0.4) are uniquely determined.

(iii) If $f \in C^{1}(\mathbb{T})$ and $\|f\|_{\infty}<1$, then also $\left\|f_{ \pm}\right\|_{\infty}<1$.

If we subsequently speak of a Blaschke representation, we always assume that $f_{+}$and $f_{-}$satisfy the conditions of assertion (i). The following lemma shows that then the right-hand side of $(\underline{0.3)}$ is well defined.

Lemma 0.2. If $f_{-} \in H_{-}^{\infty}, f_{+} \in H_{+}^{\infty}$ and $\left\|f_{ \pm}\right\|_{\infty} \leq 1$, then $1+\bar{f}_{-} f_{+} \neq 0$ almost everywhere on $\mathbb{T}$.

Proof. The function $1+\bar{f}_{-} f_{+}$is bounded on $\mathbb{T}$ and extends holomorphically into $\mathbb{D}$. If $\bar{f}_{-} f_{+}=-1$ on a subset of positive measure, then, by Privalov's uniqueness theorem, $1+\bar{f}_{-} f_{+}=0$ (almost everywhere) on $\mathbb{T}$. Since $\left|f_{ \pm}\right| \leq 1$, this would imply that $\left|f_{-}\right|=\left|f_{+}\right|=1$ and furthermore $f_{+}=-f_{-}$on $\mathbb{T}$, which is only possible if $f_{+} \equiv f_{-} \equiv 0$.

The rest of the paper is devoted to the proof of Theorem 0.1. We start with proving assertion (ii) about uniqueness of $f_{-}$and $f_{+}$.

Assume that a function $f \in L^{\infty}$ with $\|f\|_{\infty}<1$ admits two Blaschke representations, $f=f_{-} \circ f_{+}=g_{-} \circ g_{+}$. Then

$$
\left(1+\bar{f}_{-} f_{+}\right) f=f_{+}+f_{-}, \quad\left(1+\bar{g}_{-} g_{+}\right) f=g_{+}+g_{-},
$$

and subtracting these equations yields

$$
f \bar{f}_{-}\left(f_{+}-g_{+}\right)+f g_{+}\left(\bar{f}_{-}-\bar{g}_{-}\right)=f_{+}-g_{+}+f_{-}-g_{-} .
$$


Setting $\Phi_{+}:=f_{+}-g_{+}, \Phi_{-}:=f_{-}-g_{-}$, and

$$
a:=-\frac{1}{1-f \bar{f}_{-}}, \quad b:=\frac{f g_{+}}{1-f \bar{f}_{-}}
$$

results in a (formally linear) transmission problem with conjugation,

$$
\Phi_{+}=a \Phi_{-}+b \bar{\Phi}_{-} .
$$

Since $q:=\|f\|_{\infty}<1$ the functions $a$ and $b$ are bounded, Re $a \leq-c<0$, and $|b| \leq q|a|$ almost everywhere on $\mathbb{T}$. Consequently, by Corollary 4 of [5] (see also [3], Theorem 9.8 and Corollary 9.7), the homogeneous problem (0.5) has only the trivial solution, i.e., $\Phi_{+}=f_{+}-g_{+}=0$ and $\Phi_{-}=f_{-}-g_{-}=0$.

Next we prove the existence of a Blaschke representation for continuously differentiable functions with $\|f\|_{\infty}<1$. In preparing this proof we derive some auxiliary results.

For $1<p<\infty$ let $H_{ \pm}^{\infty} \cap W_{p}^{1}$ be the space of holomorphic functions in $H_{ \pm}^{\infty}$ with boundary function in the Sobolev space $W_{p}^{1}(\mathbb{T})$, equipped with the norm of the boundary functions in $W_{p}^{1}(\mathbb{T})$. Since $W_{p}^{1}(\mathbb{T})$ is (compactly) embedded in $C(\mathbb{T})$ if $p>1$, all boundary values of functions $f \in H_{ \pm}^{\infty} \cap W_{p}^{1}$ exist as usual limits.

As a first result we show that holomorphic functions in $H_{+}^{\infty} \cap W_{p}^{1}$ that map the disk into a certain sector

$$
S_{\alpha}:=\{z \in \mathbb{D}:|\arg (1-z)|<\alpha\}, \quad 0<\alpha<\pi / 2,
$$

cannot access the corner of that sector (at $z=1$ ) if $p$ is sufficiently large.

Lemma 0.3. Let $f \in H_{+}^{\infty} \cap W_{p}^{1}$ and $0<\alpha<\pi / 2$. If $f(z) \in \bar{S}_{\alpha}$ for all $z \in \mathbb{D}$ and $f(t)=1$ for some $t \in \mathbb{T}$, then $p \leq \pi /(\pi-2 \alpha)$ or $f \equiv 1$.

Proof. Assuming the contrary, we prove that $p>\pi /(\pi-2 \alpha)$ and $f \not \equiv 1$ lead to a contradiction. Without loss of generality we henceforth suppose that $f$ equals one at $t=1$.

Since $f(1)=1$ and $f \not \equiv 1$, the open mapping principle for nonconstant holomorphic functions yields that $f(z) \in S_{\alpha} \subset \mathbb{D}$ for all $z \in \mathbb{D}$. Consequently, the function $g$ defined by

$$
g(z):=\frac{1-f(z)}{1-z}, \quad z \in \mathbb{D},
$$

is holomorphic and has no zeros in $\mathbb{D}$. Moreover, $g$ belongs to the Hardy spaces $H_{+}^{r}$ for all $r \in(0,1)$ (for the definition of these spaces see, for instance, [1]) and has a continuous boundary function on $\mathbb{T} \backslash\{1\}$. By virtue of $1-f(1)=0$ and $f \in W_{p}^{1}(\mathbb{T})$ it follows from Hardy's inequality (see, for instance, 7, Chap. 1, Sect. 5.1) that the boundary function of $g$ belongs to $L^{p}(\mathbb{T})$, which, together with $g \in H_{+}^{r}$, implies that $g \in H_{+}^{p}$ (see [1, Chap. II, Cor. 4.3).

Since $f(z) \in S_{\alpha}$, i.e. $|\arg (1-f(z))| \leq \alpha$, and $|\arg (1-z)| \leq \pi / 2$, it follows that $|\arg g(z)| \leq \alpha+\pi / 2<\pi$. By the assumption on $p$, made at the beginning of the proof, there is a positive $\varepsilon$ such that

$$
p>\frac{\pi}{\pi-2 \alpha-4 \varepsilon}
$$

and we can choose $\varepsilon$ so small that $d:=\pi / 2-(\alpha+\varepsilon)>0$. If $t \in \mathbb{T}$ is close to 1 , then $\arg (1-t)$ is close to $-\operatorname{sign}(\operatorname{Im} t) \cdot \pi / 2$ and therefore we find a positive $\delta$ such 


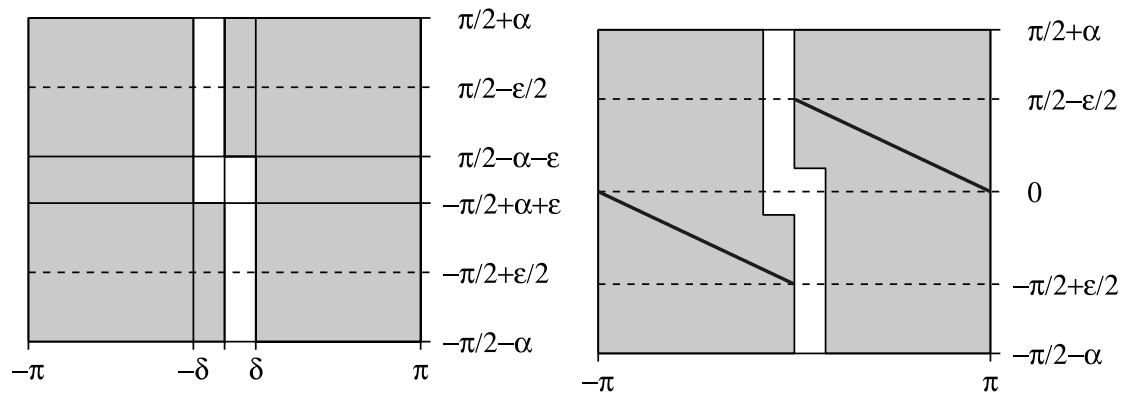

Figure 1. The arguments of $g$ and $h$

that

$$
\begin{array}{cl}
d \leq \arg g(t) \leq \alpha+\pi / 2 & \text { if } \quad t \in T_{+}:=\{t \in \mathbb{T}:|t-1| \leq \delta, \operatorname{Im} t>0\}, \\
-\alpha-\pi / 2 \leq \arg g(t) \leq-d & \text { if } \quad t \in T_{-}:=\{t \in \mathbb{T}:|t-1| \leq \delta, \operatorname{Im} t<0\},
\end{array}
$$

which implies

$$
\left|\arg g+\frac{\pi-\varepsilon}{2}\right| \leq \alpha+\frac{\varepsilon}{2} \quad \text { if } \quad t \in T_{-}, \quad\left|\arg g-\frac{\pi-\varepsilon}{2}\right| \leq \alpha+\frac{\varepsilon}{2} \quad \text { if } \quad t \in T_{+} .
$$

In summary, the graph of $\tau \mapsto \arg g\left(\mathrm{e}^{\mathrm{i} \tau}\right)$ lies in the shaded region shown in the left part of Figure 1.

In order to reduce the jump of $\arg g$ at $t=1$ we remark that the argument of the function $h$ defined by $h(t):=(1-t)^{-1+\varepsilon / \pi}$ depends linearly on the polar angle $\tau \in(0,2 \pi)$ with $\lim \arg h\left(\mathrm{e}^{\mathrm{i} \tau}\right)=(\varepsilon-\pi) / 2$ as $\tau \rightarrow 2 \pi$ and $\lim \arg h\left(\mathrm{e}^{\mathrm{i} \tau}\right)=(\pi-\varepsilon) / 2$ as $\tau \rightarrow 0$. Decreasing $\delta$, if necessary, we can ensure that

$$
\left|\arg h+\frac{\pi-\varepsilon}{2}\right| \leq \frac{\varepsilon}{2} \quad \text { if } \quad t \in T_{-}, \quad\left|\arg h-\frac{\pi-\varepsilon}{2}\right| \leq \frac{\varepsilon}{2} \quad \text { if } \quad t \in T_{+} .
$$

The graph of $\arg h$ is shown in the right part of Figure 1. The function $h g^{-1}$ is holomorphic in $\mathbb{D}$. Its boundary function is continuous on $\mathbb{T} \backslash\{1\}$ and

$$
\left|\arg \left(h(t) g^{-1}(t)\right)\right| \leq \alpha+\varepsilon \quad \text { if }|t-1| \leq \delta .
$$

Then Zygmund's Lemma (see Garnett [1, Sect. 3.3., Cor. 2.6, Koosis [2, Sect. V:D, Thm. 1, for instance) tells us that $h g^{-1} \in L^{r}$, provided $r<\pi /(2 \alpha+2 \varepsilon)$. By Hölder's inequality we obtain from $g \in L^{p}$ and $h g^{-1} \in L^{r}$ that $h \in L^{s}$ if $1 / s=1 / r+1 / p$, which is the case for all $s<\pi /(\pi-2 \varepsilon)$. This is in conflict with $h \notin L^{s}$ for $s=\pi /(\pi-\varepsilon)$.

Lemma 0.4. Let $f=f_{-} \circ f_{+} \in W_{p}^{1}(\mathbb{T})$ with $\|f\|_{\infty} \leq q<1$ and $f_{-} \in H_{-}^{\infty} \cap W_{p}^{1}$. If

$$
\frac{\pi}{\pi-2 \alpha}<p<\infty, \quad \text { with } \quad \alpha:=\arcsin \frac{2 q}{1+q^{2}},
$$

then $f_{+} \in H_{+}^{\infty} \cap W_{p}^{1}$ and $\left\|f_{ \pm}\right\|_{\infty}<1$.

Proof. We assume that $f_{-}$and $f_{+}$are not constant, because otherwise the result is trivial. Since

$$
f_{+}=\frac{f-f_{-}}{1-\bar{f}_{-} f}
$$


with $\|f\|_{\infty}<1,\left\|f_{-}\right\|_{\infty} \leq 1$, and $W_{1}^{p}(\mathbb{T})$ is an algebra, we have $f_{+} \in W_{1}^{p}(\mathbb{T})$. A direct computation of the absolute values of the Blaschke factors shows that $\left|f_{ \pm}(z)\right| \leq 1$ and $\left|f_{-}(z) \circ f_{+}(z)\right| \leq q<1$ imply that

$$
g(z):=-\overline{f_{-}(z)} f_{+}(z) \in \bar{S}_{\alpha}, \quad z \in \mathbb{D} .
$$

Assume now that $\left|f_{-}\left(t_{0}\right)\right|=1$ for some $t_{0} \in \mathbb{T}$. It can easily be seen that then $f=f_{-} \circ f_{+}$with $\|f\|_{\infty}<1$ necessarily implies $f_{+}\left(t_{0}\right)=-f_{-}\left(t_{0}\right)$ which yields $g\left(t_{0}\right)=\left|f_{-}\left(t_{0}\right)\right|^{2}=1$. Since $g$ belongs to $H_{+}^{\infty} \cap W_{p}^{1}$ with $p>\pi /(\pi-2 \alpha)$ we infer from Lemma 0.3 that $g \equiv 1$; but this is impossible by Lemma 0.2 . Finally, we conclude from the representation (0.7) and $\|f\|_{\infty}<1,\left\|f_{-}\right\|_{\infty}<1$ that also $\left\|f_{+}\right\|_{\infty}<1$.

In order to prove the existence of a Blaschke representation we first assume that $f$ belongs to $C^{1}(\mathbb{T})$ and $\|f\|_{\infty} \leq q<1$. We fix $p$ with $1<p<\infty$ such that (0.6) is satisfied and work in the Sobolev space $W_{p}^{1}(\mathbb{T})$.

Step 1. With $G$ defined by

$$
G(t, z):=\frac{f(t)-z}{1-f(t) \bar{z}}, \quad t \in \mathbb{T}, z \in \overline{\mathbb{D}},
$$

the Blaschke representation of $f$ can be rewritten as

$$
f_{+}(t)=G\left(t, f_{-}(t)\right), \quad t \in \mathbb{T} .
$$

We prove that this nonlinear Riemann-Hilbert problem (or transmission problem, see [8]) has a (unique) solution $\left(f_{+}, f_{-}\right) \in H_{+}^{\infty} \times H_{-}^{\infty}$ with $\left\|f_{ \pm}\right\|_{\infty}<1$. In preparation we remark that the derivatives

$$
\partial_{z} G(t, z)=\frac{1}{f(t) \bar{z}-1}, \quad \partial_{\bar{z}} G(t, z)=\frac{f(t)-z}{(f(t) \bar{z}-1)^{2}} f(t)
$$

are continuous on $\mathbb{T} \times \overline{\mathbb{D}}$. The derivative $\partial_{\tau} G \equiv \mathrm{i} t \partial_{t} G$ of $G$ with respect to the polar angle $\tau$ of $t \equiv \mathrm{e}^{\mathrm{i} \tau}$ is equal to

$$
\partial_{\tau} G(t, z)=\frac{1-|z|^{2}}{(f(t) \bar{z}-1)^{2}} \partial_{\tau} f(t) .
$$

This function is continuous in $z$ for almost all $t \in \mathbb{T}$ and belongs to $L^{p}(\mathbb{T})$ with respect to $t$ for all $z \in \overline{\mathbb{D}}$. Moreover, for all $(t, z) \in \mathbb{T} \times \overline{\mathbb{D}}$,

$$
-\operatorname{Re} \partial_{z} G(t, z) \geq \frac{1}{2} \quad \text { and } \quad \frac{\left|\partial_{\bar{z}} G(t, z)\right|}{\left|\partial_{z} G(t, z)\right|}=\left|\frac{f(t)-z}{1-f(t) \bar{z}}\right| \cdot|f(t)| \leq q<1 .
$$

Step 2. We construct a compact operator that reduces (0.8) to a fixed point equation. For given $f \in W_{p}^{1}(\mathbb{T})$ with $\|f\|_{\infty}<1$, the operator $K_{f}$ is defined on the closed unit ball

$$
B:=\left\{u \in C(\mathbb{T}):\|u\|_{\infty} \leq 1\right\}
$$

of $C(\mathbb{T})$. For $u \in B$ we define

$$
a(t):=\partial_{z} G(t, u(t)), \quad b(t):=\partial_{\bar{z}} G(t, u(t)), \quad c(t):=\mathrm{i} t \partial_{t} G(t, u(t)) .
$$

The functions $a$ and $b$ are continuous, while $c$ belongs to the Lebesgue space $L^{p}(\mathbb{T})$. The norms $\|a\|_{\infty},\|b\|_{\infty}$ and $\|c\|_{p}$ are bounded independently of $u$ and

$$
\frac{|b(t)|}{|a(t)|} \leq q<1, \quad-\operatorname{Re} a \geq \frac{1}{2} .
$$


In particular, the winding number of $a$ about zero vanishes. These properties guarantee that the linear transmission problem with conjugation

$$
\Phi_{+}=a \Phi_{-}+b \bar{\Phi}_{-}+c
$$

has a unique solution $\Phi_{+} \in H_{+}^{p}, \Phi_{-} \in H_{-}^{p}$ for each $p$ with $1<p<\infty$ ( $\underline{3}$, Theorem 9.8 and Corollary 9.7). Setting

$$
\widehat{\Phi}_{-}\left(\mathrm{e}^{\mathrm{i} \tau}\right):=\int_{0}^{\tau} \Phi_{-}\left(\mathrm{e}^{\mathrm{i} \sigma}\right) \mathrm{d} \sigma, \quad P_{0} \widehat{\Phi}_{-}:=\frac{1}{2 \pi} \int_{0}^{2 \pi} \widehat{\Phi}_{-}\left(\mathrm{e}^{\mathrm{i} \sigma}\right) \mathrm{d} \sigma,
$$

the operator $K_{f}: B \rightarrow W_{p}^{1}(\mathbb{T})$ is given by $K_{f}(u):=\widehat{\Phi}_{-}-P_{0} \widehat{\Phi}_{-}$. The definition of $\widehat{\Phi}_{-}$makes sense since $P_{0} \Phi_{-}=0$.

We summarize relevant properties of the operator $K$. Let $W$ be the (nonclosed) subspace

of $W_{p}^{1}(\mathbb{T})$.

$$
W:=\left\{f \in W_{p}^{1}(\mathbb{T}):\|f\|_{\infty}<1\right\}
$$

Lemma 0.5. The following assertions hold for all $p$ with $1<p<\infty$ :

(i) For all $f \in W_{p}^{1}(\mathbb{T})$ with $\|f\|_{\infty}<1$ the operator $K_{f}: B \subset C(\mathbb{T}) \rightarrow W_{p}^{1}(\mathbb{T})$ is continuous and its image is bounded.

(ii) The mapping $K: W \times B \rightarrow W_{p}^{1}(\mathbb{T}),(f, u) \mapsto K_{f}(u)$ is continuous.

Proof. To begin with we remark that the following superposition operators are continuous:

$$
\begin{aligned}
& C(\mathbb{T}) \rightarrow C(\mathbb{T}): \quad u \mapsto a:=\partial_{z} G(., u), \\
& C(\mathbb{T}) \rightarrow C(\mathbb{T}): \quad u \mapsto b:=\partial_{\bar{z}} G(., u), \\
& C(\mathbb{T}) \rightarrow L^{p}(\mathbb{T}): \quad u \mapsto c:=\mathrm{i} t \cdot \partial_{t} G(., u) .
\end{aligned}
$$

For fixed continuous functions $a$ and $b$, the transmission problem (0.10) defines a (real linear) operator

$$
T_{a, b}: H_{+}^{p} \times H_{-}^{p} \rightarrow L^{p},\left(\Phi_{+}, \Phi_{-}\right) \mapsto c:=\Phi_{+}-a \Phi_{-}-b \bar{\Phi}_{-} .
$$

The properties of $a$ and $b$ guarantee that $T_{a, b}$ is continuously invertible ([3] loc.cit.), which shows the continuity of the mapping

$$
S_{a, b}: L^{p} \rightarrow H_{-}^{p}, c \mapsto \Phi_{-} .
$$

Since $(a, b) \mapsto T_{a, b}$ is a continuous mapping of $C(\mathbb{T}) \times C(\mathbb{T})$ into the space of bounded linear operators $\mathcal{L}\left(H_{+}^{p} \times H_{-}^{p}, L^{p}\right)$, the mapping $(a, b) \mapsto S_{a, b}$ is continuous from $C(\mathbb{T}) \times C(\mathbb{T})$ into $\mathcal{L}\left(L^{p}, H_{-}^{p}\right)$, which together with the above results implies that $u \mapsto \Phi_{-}$is continuous from $C(\mathbb{T})$ into $H_{-}^{p}$. Integrating $\Phi_{-}$along $\mathbb{T}$ gives $K_{f}(u)$, and because integration is a continuous operator from $H_{-}^{p}$ into $W_{p}^{1}(\mathbb{T})$ we get the continuity claimed in assertion (i).

A similar reasoning proves the continuity statement of assertion (ii).

In order to see that the image of $K_{f}$ is bounded, we remark that the functions $a$ and $b$ defined by (0.12), (0.13) satisfy the estimates

$$
\|a\|_{\infty} \leq C, \quad\|b\|_{\infty} \leq C, \quad|b| /|a| \leq q<1
$$

with constants not depending on $u$. This implies a uniform bound on the norm of the operator $S_{a(u), b(u)}$ (see [8]) and thus on the norm of $\Phi_{-}:=S_{a, b} c$ in $H_{-}^{p}$.

Corollary 0.6. The operator $K_{f}: B \subset C(\mathbb{T}) \rightarrow C(\mathbb{T})$ is compact. 
The assertion follows from the last lemma and the compactness of the embedding $W_{p}^{1}(\mathbb{T}) \rightarrow C(\mathbb{T})$

Step 3. The next result shows in which way the functions $f_{+}$and $f_{-}$of the representation (0.3) are related to the fixed points of $K_{f}$.

Lemma 0.7. Let $f \in C^{1}(\mathbb{T})$ with $\|f\|_{\infty} \leq q<1$ and choose $p>1$ according to (0.6).

(i) If $f=f_{-} \circ f_{+}$is a Blaschke representation of $f$ and if $f_{-} \in W_{p}^{1}(\mathbb{T})$ satisfies $\left\|f_{-}\right\|_{\infty}<1$, then $f_{-}$is a fixed point of $K_{f}$.

(ii) If $u \in B$ is a fixed point of $K_{f}$, then $f_{-}:=u$ belongs to $H_{-}^{\infty} \cap W_{p}^{1},\left\|f_{-}\right\|_{\infty}<$ $1, f_{+}:=(f-u) /(1-f \bar{u})$ lies in $H_{+}^{\infty} \cap W_{p}^{1}$, and $f=f_{-} \circ f_{+}$is a Blaschke representation of $f$.

Proof. (i) If $f=f_{-} \circ f_{+}$with $f_{-} \in W_{p}^{1}(\mathbb{T})$, then also $f_{+} \in W_{p}^{1}(\mathbb{T})$. Differentiating the boundary relation $f_{+}(t)=G\left(t, f_{-}(t)\right)$ with respect to the polar angle $\tau$ we obtain that the pair $\left(\Phi_{+}, \Phi_{-}\right):=\left(\partial_{\tau} f_{+}, \partial_{\tau} f_{-}\right)$is the (unique) solution of the transmission problem (0.10). Consequently,

$$
K_{f}\left(f_{-}\right)\left(\mathrm{e}^{\mathrm{i} \tau}\right)=q+\int_{0}^{\tau} \Phi_{-}\left(\mathrm{e}^{\mathrm{i} \sigma}\right) \mathrm{d} \sigma=q+\int_{0}^{\tau} \partial_{\sigma} f_{-}\left(\mathrm{e}^{\mathrm{i} \sigma}\right) \mathrm{d} \sigma,
$$

where the constant $q$ is chosen so that the mean value of $K_{f}\left(f_{-}\right)$over $\mathbb{T}$ vanishes. The right-hand side differs from $f_{-}$at most by a constant, and since

$$
\int f_{-}\left(\mathrm{e}^{\mathrm{i} \tau}\right) \mathrm{d} \tau=2 \pi f_{-}(\infty)=0
$$

this constant must be zero, i.e. $K_{f}\left(f_{-}\right)=f_{-}$.

(ii) Now let $f_{-} \equiv u \in B$ be a fixed point of $K_{f}$ and $f_{+}:=G\left(., f_{-}\right)$. First of all, $f_{-}$and consequently $f_{+}$belong to $W_{p}^{1}(\mathbb{T})$.

The solutions $\Phi_{+}$and $\Phi_{-}$of the transmission problem (0.10) are holomorphic in $\mathbb{D}$ and $\mathbb{E}$, respectively, and $\widehat{\Phi}_{-}$defined by $(0.11)$ is holomorphic in $\mathbb{E}$. Since

$$
f_{-}=K_{f}\left(f_{-}\right)=\widehat{\Phi}_{-}+q
$$

the function $f_{-}$extends holomorphically into $\mathbb{E}$, and since the mean value of $K_{f}\left(f_{-}\right)$ over $\mathbb{T}$ vanishes by definition of $K_{f}$, we have $f_{-} \in H_{-}^{\infty} \cap W_{p}^{1}$.

The relation (0.15) implies that $\Phi_{-}=\partial_{\tau} f_{-}$, and inserting this into (0.10) shows that

$$
\Phi_{+}=a \Phi_{-}+b \bar{\Phi}_{-}+c=\partial_{\tau}\left(G\left(., f_{-}\right)\right)=\partial_{\tau} f_{+} .
$$

Consequently $\partial_{\tau} f_{+}$and hence $f_{+}$is holomorphic in $\mathbb{D}$. Finally, Lemma 0.4 tells us that $\left\|f_{ \pm}\right\|_{\infty}<1$.

Step 4. In particular, it follows from the preceding step that $K$ has no fixed points on the boundary of the ball $B$ and thus the Leray-Schauder fixed point index $\operatorname{fix}_{B} K$ of $K$ with respect to $B$ is well defined. In order to determine this index we consider the homotopy $\lambda \mapsto f_{\lambda}:=\lambda f$ with $\lambda \in[0,1]$.

With any function $f_{\lambda}$ we associate the corresponding operator $K_{\lambda f}$. The operators $K_{\lambda f}$ are compact and depend continuously on $\lambda$. Since, by Lemma 0.7 (ii), there are no fixed points of $K_{\lambda f}$ on $\partial B$, the fixed point index fix $K_{\lambda f}$ of $K_{\lambda f}$ with respect to $B$ is well defined for all $\lambda$ and, by homotopy invariance, it does not depend on $\lambda$. 
For $\lambda=0$ we have $f_{0} \equiv 0$ and $K_{0}=0$, which gives $\operatorname{fix}_{B} K_{0}=1$, so that also fix $_{B} K_{f}=1$. Hence $K_{f}$ has a fixed point in $B$, which gives rise to a Blaschke representation of $f$.

In the final part of the proof of Theorem 0.1 we drop the additional assumptions on $f$ and assume that $f \in L^{\infty}$ with $\|f\|_{\infty} \leq 1$. Then there exists a sequence $\left(f_{n}\right)$ of infinitely differentiable functions on $\mathbb{T}$ such that $\left\|f_{n}\right\|_{\infty}<1$ and $f_{n} \rightarrow f$ in $L^{2}$. For instance, let $f_{n}(t):=r P f(r t)$, where $P f$ is the harmonic extension of $f$ to $\mathbb{D}$ and $r:=1-1 / n$.

The smooth functions $f_{n}$ admit a (unique) Blaschke representation $f_{n}=f_{n}^{-} \circ f_{n}^{+}$ with $\left\|f_{n}^{ \pm}\right\|_{\infty} \leq 1$. Since the sequences $\left(f_{n}^{+}\right)$and $\left(f_{n}^{-}\right)$are also bounded in $L^{2}$ they contain subsequences converging weakly in $L^{2}$, and we can assume that $f_{n}^{+} \rightarrow f_{+}$ and $f_{n}^{-} \rightarrow f_{-}$. In order to show that $f_{n}^{-} \circ f_{n}^{+} \rightarrow f_{-} \circ f_{+}$we need the following results, which can easily be verified.

Lemma 0.8. Let $f_{n}^{ \pm} \in L^{\infty}$ and $\left\|f_{n}^{ \pm}\right\|_{\infty} \leq 1$ and assume that $f_{n}^{ \pm} \rightarrow f_{ \pm}$in $L^{2}$. Then

(i) the limits $f_{ \pm}$are in $L^{\infty}$ and $\left\|f_{ \pm}\right\|_{\infty} \leq 1$;

(ii) the product $f_{n}^{+} f_{n}^{-}$converges weakly to $f_{+} f_{-}$in $L^{2}$;

(iii) if $f_{n}^{ \pm} \in H_{ \pm}^{\infty}$, then $f_{ \pm} \in H_{ \pm}^{\infty}$.

Using these facts we take the weak limits in $f\left(1+f_{n}^{+} \bar{f}_{n}^{-}\right)=f_{n}^{+}+f_{n}^{-}$and obtain

$$
f\left(1+f_{+} \bar{f}_{-}\right)=f_{+}+f_{-} .
$$

Since $f_{-} \in H_{-}^{\infty}$, the function $1+f_{+} \bar{f}_{-}$belongs to $H_{+}^{\infty}$. So, by Privalov's uniqueness theorem, either $1+f_{+} \bar{f}_{-} \equiv 0$ or $1+f_{+} \bar{f}_{-} \neq 0$ almost everywhere on $\mathbb{T}$. The first case can be excluded, since then equation (0.16) implies that $f_{+} \equiv f_{-} \equiv 0$; in the second case $f=f_{-} \circ f_{+}$almost everywhere on $\mathbb{T}$. This completes the proof of Theorem 0.1 .

The following examples illustrate the limitations of the results. The first one shows that the Blaschke representation need not be unique if $\|f\|_{\infty}=1$.

Example 1. The function $f(t)=\bar{t}$ admits two representations $f=f_{-} \circ f_{+}=g_{-} \circ g_{+}$ with $f_{-}(z)=g_{-}(z)=1 / z, f_{+}(z)=1$, and $g_{+}(z)=z$. Indeed,

$$
f(t)=\bar{t}=\frac{1+1 / t}{1+\overline{1 / t} \cdot 1}=\frac{t+1 / t}{1+\overline{1 / t} \cdot t} \quad \text { almost everywhere on } \mathbb{T} .
$$

The second example shows that there are in fact Blaschke representations of functions $f \in L^{\infty}$ with $\|f\|_{\infty}<1$ and $\left\|f_{ \pm}\right\|_{\infty}=1$.

Example 2. If $p<1 / \lambda$ the functions $f_{+}(t)=1-(1-t)^{\lambda}$ and $f_{-}(t)=-1+(1-1 / t)^{\lambda}$ with $0<\lambda<1$ belong to $H_{ \pm}^{\infty} \cap W_{p}^{1}$ and satisfy $\left\|f_{ \pm}\right\|_{\infty}=1$. A straightforward calculation shows that $f:=f_{-} \circ f_{+}$has norm $\|f\|_{\infty}$ less than one.

Note that the function $f$ is not continuous, but has a jump of absolute value $\sin (\lambda \pi)$ at $t=1$. So this example also demonstrates that $f_{+}$and $f_{-}$can both have higher regularity than $f$. Figure 2 shows the images of the unit circle under the mappings $f, f_{+}$and $f_{-}$for $\lambda=1 / 2$.

We have no counterexample of this type where the function $f$ itself belongs to $W_{p}^{1}(\mathbb{T})$, and we conjecture that such functions do not exist.

Conjecture. Let $f \in W_{p}^{1}(\mathbb{T})$ with $1<p<\infty$. If $f$ has the Blaschke representation $f=f_{-} \circ f_{+}$and $\|f\|_{\infty}<1$, then $f_{ \pm} \in W_{p}^{1}(\mathbb{T})$ and $\left\|f_{ \pm}\right\|_{\infty}<1$. 


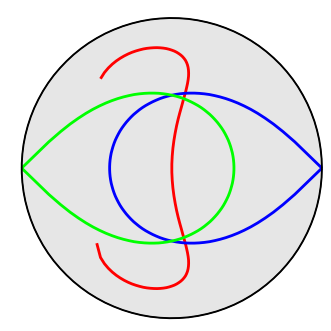

FIGURE 2 .

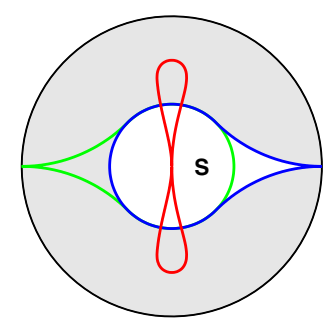

FigURE 3.

On the other hand, the third example demonstrates that the conjecture cannot be extended to continuous functions $f$.

Example 3. Let $S$ be the domain bounded by three circular arcs with centers at $1+\mathrm{i}, 1-\mathrm{i}, 0$, and radii 1,1 , and $\sqrt{2}-1$, respectively ( $S$ is the white domain in Figure $3)$. Furthermore, let $f_{+}$be the conformal mapping of $\mathbb{D}$ onto $S$, normalized so that $f_{+}(0)=0, f(1)=1$, and let $f_{-}(t):=-\bar{f}_{+}(t)$. By the Carathéodory-Osgood theorem the boundary function of $f_{+}$, and consequently of $f_{-}$, are continuous on $\mathbb{T}$. It can be easily seen that $f:=f_{-} \circ f_{+}$is continuous on $\mathbb{T}$ and satisfies $\|f\|_{\infty}<1$, while $\left\|f_{ \pm}\right\|_{\infty}=1$.

Finally, we show that the hyperbolic Parseval equation, obtained by Sylvester and Winebrenner [6] for the half-plane, also holds for the unit disc, and that it remains in force even if the energy defined by

$$
E(f):=-\int_{\mathbb{T}} \ln \left(1-|f|^{2}\right)|d t|
$$

is infinite.

Theorem 0.9. If $f=f_{-} \circ f_{+}$is a Blaschke representation of $f \in L^{\infty}$ with $\|f\|_{\infty} \leq$ 1 , then

$$
E(f)=E\left(f_{-}\right)+E\left(f_{+}\right) .
$$

Proof. By definition, the energy is always nonnegative, so the sum $E\left(f_{-}\right)+E\left(f_{+}\right)$ makes sense also if one or both summands are infinite. If $|f|=1$ on a set of positive measure, then at least one of $\left|f_{+}\right|$or $\left|f_{-}\right|$must be one on this set and both sides of (0.17) are infinite. If $|f|<1$ almost everywhere on $\mathbb{T}$, we have

$$
\left|1+\bar{f}_{-} f_{+}\right|^{2}\left(1-|f|^{2}\right)=\left|1+\bar{f}_{-} f_{+}\right|^{2}-\left|f_{+}+f_{-}\right|^{2}=\left(1-\left|f_{-}\right|^{2}\right)\left(1-\left|f_{+}\right|^{2}\right)
$$


and hence

$$
\ln \left(1-|f|^{2}\right)=\ln \left(1-\left|f_{-}\right|^{2}\right)+\ln \left(1-\left|f_{+}\right|^{2}\right)-2 \ln \left|1+\bar{f}_{-} f_{+}\right| .
$$

By the maximum principle, $\left|f_{ \pm}(z)\right|<1$ in $\mathbb{D}$. The function $g:=\log \left(1+\bar{f}_{-} f_{+}\right)$ extends holomorphically into $\mathbb{D}$ with $g(0)=0$ and its imaginary part is bounded by $\pm \pi / 2$. Thus $g$ belongs to $H^{p}$ for all $p<\infty$ and

$$
\int_{\mathbb{T}} \ln \left|1+\bar{f}_{-} f_{+}\right||d t|=\operatorname{Re} \int_{\mathbb{T}} g(t)|d t|=2 \pi \operatorname{Re} g(0)=0 .
$$

The two remaining functions on the right-hand side of (0.18) are measurable and nonpositive. So their integrals are either both finite, in which case $E(f)=E\left(f_{+}\right)+$ $E\left(f_{-}\right)<+\infty$, or at least one integral is $-\infty$, in which case $E\left(f_{+}\right)+E\left(f_{-}\right)=$ $+\infty=E(f)$.

\section{REFERENCES}

1. Garnett, J.B.: Bounded Analytic Functions. Academic Press, 1981. MR628971 (83g:30037)

2. Koosis, P.: Introduction to $H_{p}$ spaces (2nd edition). Cambridge University Press, 1998. MR:565451 (81c:30062)

3. Litvinchuk, G.M., and I.M. Spitkovsky: Factorization of Measurable Matrix Functions. Birkhäuser, Basel, 1987. MR 1015716 (90g:47030)

4. Mityushev, V.V., and S.V. Rogosin: Constructive Methods for Linear and Nonlinear Boundary Value Problems for Analytic Functions. Chapman and Hall/CRC Press, 2000. MR1739063 (2001d:30075)

5. Spitkovsky, I.M.: On the theory of the generalized Riemann boundary value problem in $L_{p}$ spaces (Russian). Ukrain. Mat. J. 31, 1 (1979) 63-73. MR523157(81e:30053)

6. Sylvester, J., and D.P. Winebrenner: Linear and nonlinear inverse scattering. SIAM J. Appl. Math. 59, No.2, 669-699 (1999). MR1654391 (2000a:34021)

7. Triebel, H.: The Structure of Functions. Birkhäuser, 2001. MR.1851996 (2002k:46087)

8. Wegert, E., Khimshiashvili, G., Spitkovsky, I.: Nonlinear transmission problems. Mem. Differ. Equ. Math. Phys. 12, 223-230 (1997). MR1636880 (99g:47058)

Institute of Applied Analysis, TU Bergakademie Freiberg, 09596 Freiberg, Germany

E-mail address: wegert@math.tu-freiberg.de

Institute of Applied Analysis, TU Bergakademie Freiberg, 09596 Freiberg, Germany E-mail address: wolfersd@math.tu-freiberg.de 